Journal of

Accident and

Emergency

Medicine 1995

12, 151-153

\title{
Orbital cellulitis demands early recognition, urgent admission and aggressive management
}

\author{
D.M.TOLE, L.C. ANDERTON \& J.M.HAYWARD
}

Department of Opthalmology, Clarendon Wing, Leeds General Infirmary, Belmont Grove, Leeds

\section{INTRODUCTION}

Orbital cellulitis is an emergency. Confusion still exists between the diagnosis of this serious condition and that of preseptal cellulitis. Delay in treatment may cause blindness and progression to life-threatening sequelae such as brain abscess, meningitis or cavernous sinus thrombosis. We report a case in which, despite late referral, emergency surgical intervention was sight saving.

Key words: orbital cellulitis

\section{CASE HISTORY}

A 79-year-old man was referred to the accident and emergency (A\&E) department with a 5-day history of left periorbital swelling, discharge and reduced visual acuity. He had been treated at home with oral cephradine $500 \mathrm{mg} 6$ hourly for $48 \mathrm{~h}$ prior to admission. His past medical history was unremarkable. He was allergic to penicillin. On examination the visual acuities were right: $6 / 5$ and left: counting fingers only. A left relative afferent pupillary defect (RAPD) was present. There was marked periorbital erythema and oedema with gross proptosis. The eye itself was red with conjunctival chemosis and the intraocular pressure normal. The fundus could not be visualized and there was marked restriction of ocular movements. The patient was afebrile with a normal white cell count.

Computerized tomography (CT) examination (Fig. 1) confirmed severe proptosis with ill-defined soft tissue shadowing within the orbit. There was intraorbital gas and fluid levels in both maxillary antra, left ethmoidal and left frontal sinuses (Fig. 2).

Approximately $4 \mathrm{~h}$ after presentation through the hospital's main A\&E department there was a further deterioration in his visual acuity to perception of hand movements, only in the left eye. At this stage an immediate lateral canthotomy was performed under local anaesthesia, in an attempt to lower the intraorbital pressure.

One and a half hours later surgery was performed under general anaesthesia by a combined ophthalmological and otorhinolaryngological team. The orbital abscess was drained via an inferior transeptal approach. Antral washouts were performed and posterior nasal and ethmoidal polyps avulsed. Copious amounts of pus were released, after which the globe could easily be retropulsed. The surgical procedure was covered with intravenous cefotaxime and metronidazole which were continued postoperatively. The antral washings grew Haemophilus influenzae which was sensitive to cefotaxime and ampicillin.

Following surgery the patient's condition improved slowly. On the second post-operative day his vision had improved to $6 / 36$. The fundus showed a slightly swollen disc with venous engorgement but no evidence of central retinal vein or artery occlusion.

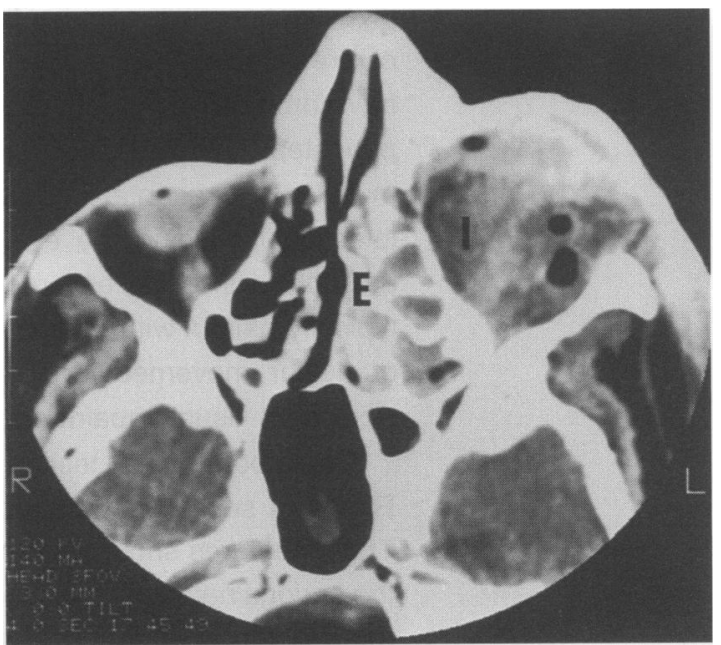

Fig. 1. CT scan orbit. Note marked proptosis, inflammatory mass, and gas locules (I) with adjacent ethmoidal sinusitis (E). 
D.M. Tole et al.

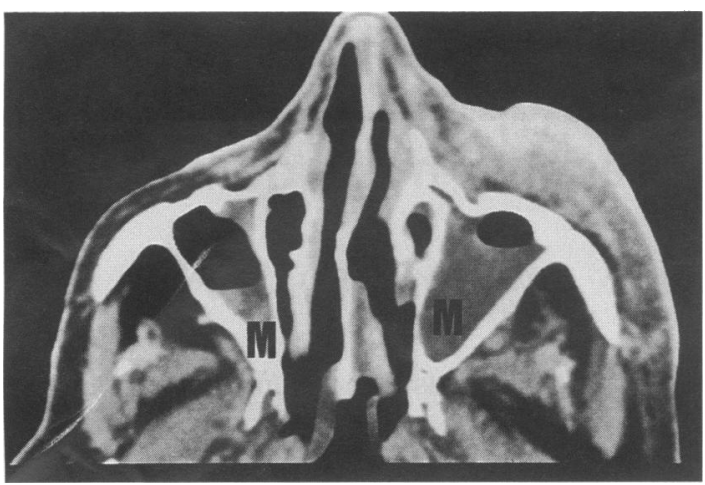

Fig. 2. CT scan orbit demonstrating fluid levels in the maxillary antra $(M)$.

One week after surgery his visual acuity had improved to 6/9 although a subtle left RAPD persisted. After a week of intravenous treatment oral antibiotics were substituted and continued for a futher 5 days. One month later his visual acuity remained at $6 / 9$ with resolution of the relative afferent pupillary defect. The eye movements and optic disc apearance were normal. His only problems were left infra-orbital paraesthesia and a 3-mm ptosis due to levator palpebrae superioris disinsertion.

\section{DISCUSSION}

The distinction between orbital and preseptal cellulitis is often difficult to make on clinical grounds alone. The investigation of choice is CT scanning of the orbits which will demonstrate the extent of inflammation, identify an abscess and show coexistent pathology within the sinuses. ${ }^{1}$ In a recent investigation, $30 \%$ of patients with the clinical finding of preseptal cellulitis were shown on CT scan to have orbital involvement (periosteal elevation, subperiosteal fluid collection, or enlarged extraocular muscles). ${ }^{2}$ Preseptal cellulitis is characterized by infection limited to the eyelid. Orbital cellulitis, in addition to swelling and redness of the eyelids, is typically painful with chemosis, proptosis, limitation of ocular movements and often associated with significant visual impairment.

The importance of testing visual acuity and for a RAPD in such cases cannot be overemphasized. These features should alert medical practitioners to the serious nature of the condition and prompt early referral for specialist treatment. The organisms most commonly responsible for orbital cellulitis are Staphylococcus aureus, Streptococcus pnue-
Acute bacterial sinusitis is present in $60-80 \%$ of cases. $^{4-6}$ The spread of infection from sinuses to the orbit by direct invasion or haematogenous spread is facilitated by the relatively thin orbital bones. ${ }^{7}$ Orbital cellulitis with gas forming organisms has been reported, ${ }^{8,9}$ however in this case the orbital gas resulted from a direct communication between the maxillary antrum and the orbital cavity (noted during the antral washouts).

The raised intraorbital pressure in orbital cellulitis results from the inflammatory oedema and pus within the bony confines of the orbit. This causes the rapid development of optic nerve compression and ischaemia, the hallmarks of this being reduced visual acuity, RAPD and impaired colour vision. The optic nerve may also be involved directly by contiguous inflammation of its dural coat. Irreversible damage can occur very rapidly, so that in the presence of orbital cellulitis with optic nerve dysfunction urgent surgical decompression is mandatory.

Our patient was fortunate to recover good vision despite severe optic nerve dysfunction, as late referral is frequently associated with blindness. ${ }^{10} \mathrm{~A}$ high index of suspicion and a low threshold for early specialist referral is required for the optimal management of orbital cellulitis.

\section{ACKNOWLEDGEMENTS}

We should like to thank Mrs Helen Cruickshank FRCS for her help with the surgical management of this case.

\section{REFERENCES}

1. Rootman J. (1988) Diseases of the Orbit, pp. 143-55. J.B. Lippincott, Philadelphia.

2. Foster J.A., Nguyen Q. \& Ratowitz J.A. (1993) is all preseptal cellulitis really preseptal? Ophthalmology 100(9a), 118.

3. Schramm V., Myers E. \& Kennerdell J. (1978) Orbital complications of acute sinusitis: evaluation, management and outcome. Otolaryngology 86, 221-230.

4. Bergin D.J. \& Wright J.E. Orbital cellulitis. (1986) British Journal of Ophthalmology 70, 174-178.

5. Amies D.R. Clinical records Orbital cellulitis. (1974) Journal of Laryngolotology 88, 559-564.

6. Schramm V., Curtin H. \& Kennerdell J. (1982) Evaluation of orbital cellulitis and results of treatment. Laryngoscope 92, 732-738.

7. Duke-Elder S. (1961) System of Ophthalmology. Vol. 2, p.479. Kimpton, London.

8. Rose G.E., Hadley J., Morgan D. \& Thompson P. (1992) Acute orbital cellulitis due to gas forming 
bacteria. Eye 5, 640-641.

9. Sevel D., Tobiens B. \& Sellars S.L., Forder A. (1973)

Gas in the orbit associated with orbital cellulitis and paranasal sinusitis. British Journal of Ophthalmology
157, $133-137$.

10. Hodges E. \& Tabbara K.F. Orbital cellulitis: review of 23 cases from Saudi Arabia. (1989) British Journal of Ophthalmology 73(3), 205-208. 